\title{
PAPER
}

\section{$\left.\left[{ }^{123}\right]\right] \beta-C I T$ SPECT is a useful method for monitoring dopaminergic degeneration in early stage Parkinson's disease}

\author{
A Winogrodzka, P Bergmans, J Booii, E A van Royen, J C Stoof, E C Wolters
}

See editorial commentary, pages 287
Objectives: To examine the validity of $\left[{ }^{123}\right]$ B-CIT SPECT for monitoring the progression of dopaminergic degeneration in Parkinson's disease; to investigate the influence of short term treatment with $D_{2}$ receptor agonists on striatal $\left[{ }^{123} \mid\right] \beta-C I T$ binding; and to determine the sample size and frequency of SPECT imaging required to demonstrate a significant effect of a putative neuroprotective agent.

Methods: A group of 50 early stage Parkinson's disease patients was examined. Two SPECT imaging series were obtained, 12 months apart. The mean annual change in the ratio of specific to non-specific $\left[{ }^{123} I\right] \beta-C I T$ binding to the striatum, putamen, and caudate nucleus was used as the outcome measure. Results: A decrease in $\left[{ }^{123} \mid\right] \beta-$-CIT binding ratios between the two images was found in all regions of interest. The average decrease in $\left[{ }^{123} \mid\right] \beta$-CIT binding ratios was about $8 \%$ in the whole striatum, $8 \%$ in the putaminal region, and $4 \%$ in the caudate region. Comparison of scans done in nine patients under two different conditions-in the off state and while on drug treatment-showed no significant alterations in the expression of striatal dopamine transporters as measured using [ $\left.{ }^{123} \mid\right] \beta$-CIT SPECT. Power analysis indicated that to detect a significant $(p<0.05)$ effect of a neuroprotective agent with 0.80 power and $30 \%$ of predicted protection within two years, 216 patients are required in each group when the effects are measured in the whole putamen.

Conclusions: $\left[{ }^{123}\right]$-CIT SPECT seems to be a useful tool to investigate the progression of dopaminergic degeneration in Parkinson's disease and may provide an objective method of measuring the effectiveness of neuroprotective treatments. Short term treatment with a $D_{2}$ agonist does not have a significant influence on $\left[{ }^{123} \mid\right] \beta$-CIT binding to dopamine transporters. If the latter finding is replicated in larger groups of patients, it supports the suitability of $\left[{ }^{123}\right] \beta$-CIT SPECT for examining the progression of neurodegeneration in patients being treated with $D_{2}$ receptor agonists.
$\mathrm{T}$ he pathophysiological hallmark of Parkinson's disease is a slow, progressive degeneration of dopaminergic neurones in the substantia nigra. ${ }^{1}$ Standard therapeutic interventions are aimed at replenishing empty dopamine stores with levodopa, or substitution with dopamine receptor agonists. However, in the long term this symptomatic treatment fails. Various neuroprotective agents are currently being developed with the intention of treating the cause of the disease and thereby delaying the degeneration of dopaminergic neurones. To evaluate the effectiveness of such agents, it is important to develop methods that can reliably measure progression of dopaminergic degeneration. Positron emission tomography (PET) and single photon emission computed tomography (SPECT) could prove to be objective tools for measuring the effectiveness of putative neuroprotective agents and monitoring disease progression. For example, recent studies using $\left[{ }^{18} \mathrm{~F}\right]$ dopa PET have shown that the presynaptic dopaminergic degeneration in Parkinson's disease is faster than in normal aging..$^{2-4}$

Since 1993, $\left[{ }^{123} \mathrm{I}\right] \beta$-CIT SPECT has been used to investigate the presynaptic dopaminergic system in Parkinson's disease by assessing the concentration of striatal dopamine transporters. Several studies have shown that decreased striatal $\left[{ }^{123} \mathrm{I}\right] \beta$ CIT binding correlates well with symptom severity. ${ }^{56}\left[{ }^{123} \mathrm{I}\right] \beta$ CIT SPECT is now considered a highly reproducible technique which could be of value in monitoring the progression of dopaminergic degeneration in Parkinson's disease. ${ }^{78}$

Our aim in this study was to investigate whether serial $\left[{ }^{123} \mathrm{I}\right] \beta$-CIT SPECT imaging can be used as a marker of Parkinson's disease progression. We undertook two $\left[{ }^{123} \mathrm{I}\right] \beta$-CIT SPECT imaging series 12 months apart in a group of 50 patients with early stage Parkinson's disease. We also estimated the sample size and scan interval necessary to predict the effectiveness of a putative neuroprotective agent.

\section{METHODS \\ Subjects}

For the "progression study" a group of 50 patients with Parkinson's disease ( 31 men and 19 women) was examined by clinical assessment and $\left[{ }^{123} \mathrm{I}\right] \beta$-CIT SPECT imaging. The patients were recruited from the movement disorders unit of our outpatient clinic. The diagnosis of Parkinson's disease was established according to the UK Parkinson's Disease Society brain bank criteria. ${ }^{9}$ The mean (SD) age of the patients at the time of the first imaging was 56.7 (9.3) years, range 37 to 71. The mean duration of Parkinson's disease was 2.7 (2.2) years. The Hoehn and Yahr staging scale ${ }^{10}$ and the unified Parkinson's disease rating scale (UPDRS) ${ }^{11}$ were used to assess the stage and severity of the disease at the time of first imaging. A detailed clinical and demographic description is given in table 1. Each patient was imaged on two occasions, with a mean scan to scan interval of 51 (7) weeks.

All patients gave written informed consent to the research protocol, which was approved by the medical ethics committee of the hospital.

\section{Drug treatment}

All patients in the "progression study" were drug-naive at the time of the first scan, after which dopaminergic treatment (levodopa or $\mathrm{a}_{2}$ receptor agonist) was initiated.

A previous study in patients with Parkinson's disease showed no significant effects of subchronic administration of 
Table 1 Clinical and demographic data on 50 patients with Parkinson's disease

\begin{tabular}{ll}
\hline Male/female & $31 / 19$ \\
Age at first imaging (years) & $56.7(9.3)$ \\
Age at onset (years) & $54.0(9.8)$ \\
Duration of disease (years) & $2.7(2.2)$ \\
Hoehn and Yahr stage & $1.9(0.6)$ \\
UPDRS motor score & $19.2(6.7)$ \\
\hline
\end{tabular}

Data are $\mathrm{n}$ or mean (SD).

UPDRS, unified Parkinson's disease rating scale.

levodopa or L-selegiline on striatal $\left[{ }^{123} \mathrm{I}\right] \beta$-CIT binding to dopamine transporters. ${ }^{12}$ Likewise, $\mathrm{D}_{2}$ receptor agonists appear to have no influence on the expression of the striatal dopamine transporter. ${ }^{13-15}$ However, the effects of these drugs on striatal $\left[{ }^{123} \mathrm{I}\right] \beta$-CIT binding in Parkinson's disease have only been examined in one study. ${ }^{28}$

Effect of $D_{2}$ receptor agonist on striatal $\left[{ }^{123} \mid\right] \beta-C I T$ binding In order to assess the effects of $\mathrm{D}_{2}$ receptor agonists on striatal $\left[{ }^{123} \mathrm{I}\right] \beta$-CIT binding in our study, nine patients with Parkinson's disease (seven male and two female; age 61.5 (8.2) years; duration of Parkinson's disease 3.2 (1.5) years) were imaged twice: first, when not previously exposed to $\mathrm{D}_{2}$ receptor agonist treatment or when withdrawn from it, and second, while on treatment with a $\mathrm{D}_{2}$ receptor agonist. The scan to scan interval was two to five weeks.

Six of the patients had not previously received $\mathrm{D}_{2}$ receptor agonist treatment at the time of the first imaging. In these six patients (protocol $\mathrm{A}$ ), treatment with a $\mathrm{D}_{2}$ receptor agonist (pergolide or pramipexol) was started one day after the baseline imaging, following an increasing schedule up to 2.0 $\mathrm{mg} /$ day for pergolide and $2.25 \mathrm{mg} /$ day for pramipexol (table 2 ). In these patients, the second imaging was done after four to five weeks while they were on treatment with the $\mathrm{D}_{2}$ receptor agonist. In the last week before the second imaging, the daily dose of the $\mathrm{D}_{2}$ receptor agonist was maintained stable.

The other three patients in the $\mathrm{D}_{2}$ receptor agonist assessment study were first imaged while on a stable dose of pergolide, which had been given as monotherapy for about a year. After the initial imaging, the drug was withdrawn for two to three weeks and the second imaging was done (protocol B).

The effects of $\mathrm{D}_{2}$ receptor agonist treatment on $\left[{ }^{123} \mathrm{I}\right] \beta$-CIT binding measures and the treatment details of the patients are summarised in table 2 .

\section{SPECT camera}

The Strichman Medical Equipment 810X system (Strichman Inc, Medfield, Massachasetts, USA) was used for SPECT imaging. The transaxial resolution of this camera is $7.6 \mathrm{~mm}$ full width at half maximum of a line source in air. ${ }^{16}$ The energy window was set at 135-190 keV. Data acquisition took place in a $128 \times 128$ matrix.

\section{SPECT imaging}

The patients were given potassium iodide orally to block the thyroid uptake of free radioactive iodide. $\left[{ }^{123} \mathrm{I}\right] \beta$-CIT (specific activity > $185 \mathrm{MBq} / \mathrm{nmol}$; radiochemical purity > 99\%) was injected intravenously at an approximate dose of $110 \mathrm{MBq} \cdot{ }^{123} \mathrm{I}$ labelling, acquisition, attenuation correction, and reconstruction of images was done as described before. ${ }^{6}$ The measured concentration of radioactivity was expressed as Strichman medical units (SMUs; I SMU $=100 \mathrm{~Bq} / \mathrm{ml}$ ).

Image acquisition was always started 24 hours after the injection of the radioligand. Slices were acquired during $300 \mathrm{~s}$ periods after positioning the patient's head in the camera, with beams from gantry mounted lasers oriented parallel to the canthomeatal (CM) line, from the CM line to the vertex using an interslice distance of $10 \mathrm{~mm}$. Imaging was always done using the same equipment and following the same protocol.

During the second image session, all efforts were made to ensure that the patient's head in the camera conformed to the position used during the first image session. To achieve this, the distances from the meatuses of the ears and from the orbital angles to the position of the laser beams were recorded. In previous studies we showed that this procedure is highly reproducible. ${ }^{17}$

\section{Data processing}

For analysis of striatal $\left[{ }^{123} \mathrm{I}\right] \beta$-CIT binding, two transverse slices representing the most intense striatal binding were summed. A standard region of interest (ROI) template, constructed according to a stereotactic atlas and including fixed regions for caudate nucleus, putamen, whole striatum, and occipital cortex, was placed bilaterally on the combined image, as described previously. ${ }^{16}$ Estimates of specific striatal binding were made by subtracting occipital counts (nonspecific binding) from total striatal counts. The ratio of specific to non-specific striatal $\left[{ }^{123} \mathrm{I}\right] \beta$-CIT binding was then calculated by dividing the specific striatal uptake by the occipital uptake. These binding ratios were also used to calculate comparable ratios for caudate nucleus and putamen. ${ }^{18}$

\section{Statistics}

The relation between the initial $\left[{ }^{123} \mathrm{I}\right] \beta$-CIT binding ratios and UPDRS was measured using the Spearman rank correlation. A paired samples two tailed $t$ test was used to examine the change between baseline and follow up imaging results. The mean annual rate of decline in $\left[{ }^{123} \mathrm{I}\right] \beta$-CIT binding ratios was

Table 2 Effects of treatment with $D_{2}$ agonists on ratios of specific to non-specific $\left[{ }^{123} \mid\right] \beta-C I T$ binding in the whole striatum

\begin{tabular}{llllll}
\hline \multirow{2}{*}{$\begin{array}{l}\text { Patient } \\
\text { No }\end{array}$} & & & & \multicolumn{2}{l}{ Striatal [123] $\beta-C I T$ binding ratio } \\
\cline { 5 - 5 } & Sex & Protocol & Drug (mg/day) & On treatment & Drug-naive/withdrawn \\
\hline 1 & F & B & Pergolide (1.0) & 1.72 & 1.79 \\
2 & M & B & Pergolide (2.0) & 1.95 & 2.30 \\
3 & M & B & Pergolide (2.0) & 1.63 & 1.66 \\
4 & M & A & Pergolide (2.0) & 2.13 & 1.88 \\
5 & F & A & Pramipexol (2.25) & 2.86 & 2.49 \\
6 & M & A & Pergolide (1.5) & 1.86 & 2.10 \\
7 & M & A & Pergolide (1.5) & 3.25 & 3.78 \\
8 & M & A & Pergolide (2.0) & 2.22 & 2.22 \\
9 & M & A & Pergolide (1.0) & 2.70 & 2.90 \\
& & & Mean (SD): & $\mathbf{2 . 2 6}(\mathbf{0 . 6})$ & $\mathbf{2 . 3 5}(\mathbf{0 . 7 0 )}$ \\
\hline
\end{tabular}

$\mathrm{F}$, female; $M$, male; protocol $A$, drug-naive at the time of first imaging; protocol $B$, approximately one year on monotherapy at the time of first imaging. 

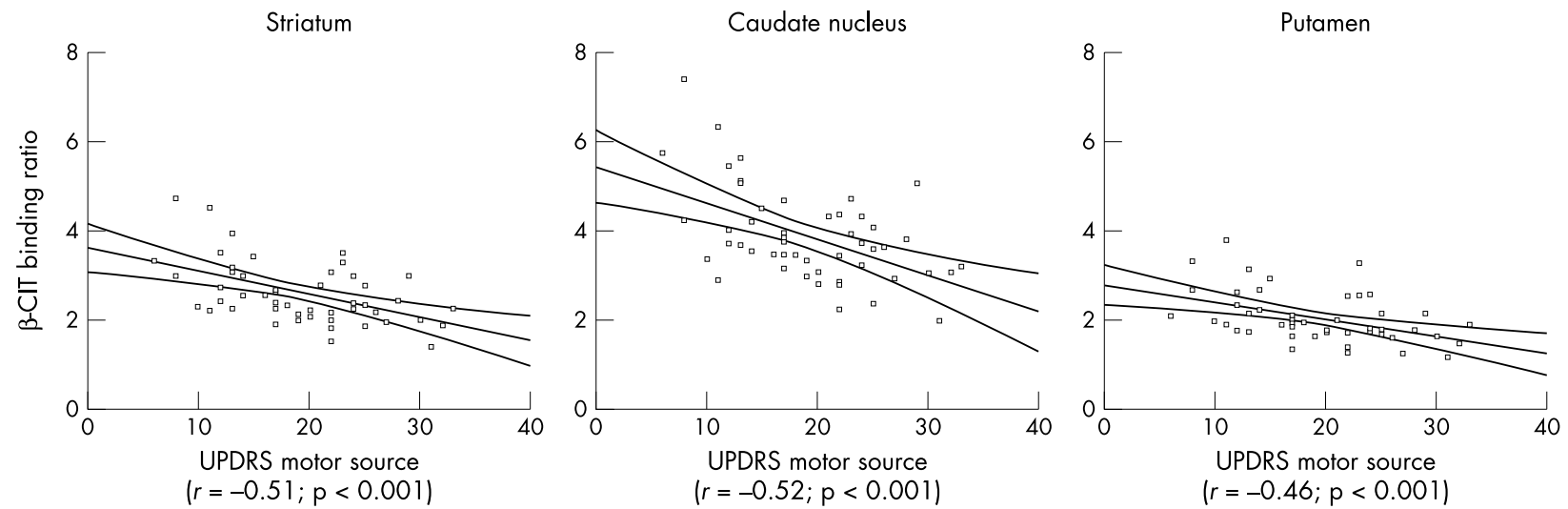

Figure 1 Correlation (Spearman rank) of the unified Parkinson's disease rating scale (UPDRS) motor score with specific to non-specific $\left.\left[{ }^{123}\right]\right] \beta$-CIT binding in striatum, putamen, and caudate nucleus in 50 patients with Parkinson's disease.

expressed as a percentage of the baseline $\left[{ }^{123} \mathrm{I}\right] \beta$-CIT binding ratios, and was calculated for each patient using the following formula:

[follow up SPECT imaging minus baseline SPECT imaging]/[baseline SPECT imaging] $\times 100 \%$

For the assessment of the effects of $\mathrm{D}_{2}$ receptor agonists on $\left[{ }^{123} \mathrm{I}\right] \beta$-CIT binding, the two conditions (on drug treatment, and either withdrawn from drug treatment or drug-naive) were compared using the Wilcoxon signed ranks test.

Power analysis was undertaken to estimate the sample size and the scan interval required to demonstrate a significant neuroprotective effect of agents with various degrees of predicted protection. The analysis assumed an annual rate of dopaminergic degeneration based on the data obtained in the present study. The standard deviation of the mean annual change in $\left[{ }^{123} \mathrm{I}\right] \beta$-CIT binding was used as a measure of variance. Sample size was determined using Altman's nomogram. ${ }^{19}$

Significance was assumed at a probability $(p)$ value of $<0.05$. In the case of multiple comparisons the Bonferroni correction was used.

\section{RESULTS}

No significant difference in age, disease severity, or $\left[{ }^{123} \mathrm{I}\right] \beta$-CIT binding measures was found between male and female subjects.

\section{Progression of dopaminergic degeneration}

The baseline ratios of specific to non-specific $\left[{ }^{123} \mathrm{I}\right] \beta$-CIT binding in all striatal regions of interest showed significant correlations with baseline motor UPDRS score $\left(r_{\mathrm{s}}\right.$ values varying from -0.32 to $-0.5 ; \mathrm{p}<0.02$; fig 1$)$. A decrease in $\left[{ }^{123} \mathrm{I}\right] \beta$-CIT binding ratios between the two consecutive scans was found in all regions of interest (table 3). There was a significant decrease in specific to non-specific $\left[{ }^{123} \mathrm{I}\right] \beta$-CIT binding ratios in the whole, ipsilateral, and contralateral striatum and putamen, this being most obvious in the whole putamen. The relative annual rate of decrease in $\left[{ }^{123} \mathrm{I}\right] \beta$-CIT binding ratios was about $8 \%$ in striatal regions, $8 \%$ in putaminal regions, and $4 \%$ in caudate regions.

No correlation was found between the rate of progression in all regions of interest and the baseline binding ratios, the duration of Parkinson's disease symptoms, or the severity of the disease expressed by the UPDRS motor score.

Effect of $D_{2}$ receptor agonist on striatal $\left[{ }^{123} I\right] \beta-C I T$ binding Treatment with $\mathrm{D}_{2}$ receptor agonists did not cause significant changes in the striatal $\left[{ }^{123} \mathrm{I}\right] \beta$-CIT binding ratios, as measured by serial SPECT imaging under the two different conditions
Table 3 Mean annual change in the ratio of specific to non-specific $\left[{ }^{123}\right.$ I] $\beta$-CIT binding in 50 patients with early stage Parkinson's disease

\begin{tabular}{llll}
\hline & $\begin{array}{l}\text { Baseline } \\
\text { ratio }\end{array}$ & $\begin{array}{l}\text { Follow up } \\
\text { ratio }\end{array}$ & Relative change \\
\hline Striatum, whole & $2.6(0.7)$ & $2.4(0.7)$ & $-7.6(17.9)^{*}$ \\
Putamen, whole & $2.1(0.6)$ & $1.9(0.5)$ & $-8.4(18.6)^{*}$ \\
Caudate, whole & $3.9(1.0)$ & $3.7(1.1)$ & $-4.5(21.3)$ \\
Striatum, ipsilateral & $2.9(0.8)$ & $2.6(0.8)$ & $-7.5(17.6)^{*}$ \\
Striatum, contralateral & $2.3(0.6)$ & $2.1(0.7)$ & $-7.1(20.7)^{*}$ \\
Putamen, ipsilateral & $2.4(0.7)$ & $2.2(0.7)$ & $-8.0(17.9)^{*}$ \\
Putamen, contralateral & $1.7(0.5)$ & $1.6(0.5)$ & $-7.9(25.2)^{*}$ \\
Caudate, ipsilateral & $4.2(1.1)$ & $4.0(1.2)$ & $-4.4(21.2)$ \\
Caudate, contralateral & $3.6(1.0)$ & $3.4(1.1)$ & $-4.5(22.8)$ \\
\hline
\end{tabular}

Values are mean (SD).

The relative change in [ $\left.{ }^{123}\right][\mathrm{B}$-CIT binding ratios within one year was calculated using the formula: [follow up SPECT imaging minus baseline SPECT imaging]/[baseline SPECT imaging] $\times 100 \%$ * Significant relative difference between the mean values of two imaging assessments (paired samples two tailed $t$ test; $p<0.05$ ).

(protocol A and protocol B) described above: on treatment $v$ drug naive or withdrawn from drugs, $2.26(0.6) v 2.35$ (0.67); $\mathrm{p}=0.4$; table 2 ).

\section{Power analysis}

Power analysis indicated that in order to detect a significant effect of a neuroprotective agent with 0.80 power and $50 \%$ of predicted protection within two years, 78 patients would be required in each group when the effects are measured by means of changes in $\left.{ }^{123} \mathrm{I}\right] \beta$-CIT binding ratios in the whole putamen. For a trial with an agent with $30 \%$ of predicted neuroprotection, 216 patients would be required. Assuming a linear decline in dopaminergic function, it was calculated that extending the scanning interval to five years reduces the required sample size to 13 patients in each group for a trial with an agent with $50 \%$ of predicted protection, and to 35 patients in each group for a trial with an agent with $30 \%$ of predicted protection (table 4).

\section{DISCUSSION}

This is one of the few $\left[{ }^{123} \mathrm{I}\right] \beta$-CIT SPECT studies done so far to investigate the value of the technique in monitoring the progression of Parkinson's disease by serial evaluation in a large group of patients with early stage disease..$^{20-22}$

In agreement with other studies, we found significant correlations between $\left[{ }^{123} \mathrm{I}\right] \beta$-CIT binding ratios and UPDRS motor score in drug-naive patients. ${ }^{56}$ The binding ratios obtained at the first series of imagings were approximately $35 \%$ of control for the putamen and $55 \%$ of control for the caudate nucleus. ${ }^{23}$ 
Table 4 Sample size and scan to scan interval required to give $80 \%$ power of detecting a significant $(p<0.05)$ effect of a neuroprotective agent, with predicted protection of $50 \%$ and $30 \%$ (assuming the reported relative rate of dopaminergic degeneration, in each region separately)

\begin{tabular}{lllllll}
\hline \multicolumn{7}{l}{ Time interval } \\
\hline & \multicolumn{2}{l}{ One year protection } & \multicolumn{2}{l}{ Two years protection } & \multicolumn{2}{l}{ Five years protection } \\
\hline Region of interest & $50 \%$ & $30 \%$ & $50 \%$ & $30 \%$ & $50 \%$ & $30 \%$ \\
\hline Striatum, whole & 340 & 949 & 85 & 238 & 14 & 38 \\
Striatum, ipsilateral & 346 & 961 & 87 & 241 & 14 & 39 \\
Striatum, contralateral & 534 & 1483 & 134 & 371 & 22 & 60 \\
Putamen, whole & 310 & 863 & 78 & 216 & 13 & 35 \\
Putamen, ipsilateral & 312 & 866 & 79 & 217 & 13 & 35 \\
Putamen, contralateral & 636 & 1775 & 160 & 444 & 26 & 71 \\
Caudate, whole & 1395 & 3851 & 349 & 963 & 56 & 155 \\
Caudate, ipsilateral & 1485 & 4112 & 372 & 1028 & 60 & 165 \\
Caudate, contralateral & 1641 & 4545 & 411 & 1137 & 66 & 182 \\
\hline
\end{tabular}

These results are in line with the findings of previous studies, ${ }^{5-6}$ indicating that the $\left[{ }^{123} \mathrm{I}\right] \beta$-CIT SPECT technique is a sensitive marker of disease severity in Parkinson's disease.

The test/retest reproducibility of $\left[{ }^{123} \mathrm{I}\right] \beta$-CIT SPECT has also recently been investigated by Seibyl et al. ${ }^{7}$ These investigators showed that $\left[{ }^{123} \mathrm{I}\right] \beta$-CIT SPECT imaging is a highly reproducible measure of striatal dopamine transporters in Parkinson's disease and suggested that the method would be suitable for the serial evaluation of dopaminergic degeneration.

In the present study, we showed that the mean annual rate of dopaminergic degeneration in striatum, and subregionally in the putamen, reached statistical significance at about $8 \%$. These findings fit well with the results of recent PET and SPECT studies. ${ }^{412425}$ For example, Morrish et al and the Parkinson Study Group reported mean annual rates of dopaminergic degeneration in Parkinson's disease of 9\% and $5 \%$, respectively. ${ }^{41}$ In addition, Van Dyck et al investigated age related changes in dopamine transporter binding with $\left[{ }^{123} \mathrm{I}\right] \beta$ CIT SPECT in human controls ${ }^{26}$ and found approximately an $8 \%$ decline per decade. In this context, our results show that the rate of progression of dopaminergic degeneration is much faster in Parkinson's disease than in normal aging.

The mean rate of progression of the dopaminergic degeneration in the caudate was slower than in the putamen. This is in agreement with the results of cross sectional SPECT and PET studies, indicating that the function of the caudate nucleus in the early Parkinson's disease stages is relatively spared.

Though the patients in the present study were under dopaminergic drug treatment (levodopa or a $\mathrm{D}_{2}$ agonist) at the time of the second imaging, Laruelle et al showed that infusion of high dose levodopa failed to displace striatal $\left[{ }^{123} \mathrm{I}\right] \beta$-CIT binding in non-human primates. ${ }^{27}$ In line with this observation, Innis et al demonstrated recently that treatment with levodopa or L-selegiline in Parkinson's disease causes neither significant occupancy nor modulation in the number of striatal dopamine transporters labelled with $\left[{ }^{123} \mathrm{I}\right] \beta$-CIT. ${ }^{12}$ Moreover, Ahlskog and coworkers ${ }^{28}$ reported that short term treatment with the dopamine $\mathrm{D}_{2}$ agonist pergolide did not significantly influence binding of $\left[{ }^{123} \mathrm{I}\right] \beta$-CIT to dopamine transporters in Parkinson's disease.

In order to assess the effects of $\mathrm{D}_{2}$ agonists on $\left[{ }^{123} \mathrm{I}\right] \beta$-CIT binding to the dopamine transporters we also undertook sequential imaging in nine patients with Parkinson's disease under two different conditions: on $\mathrm{D}_{2}$ agonist treatment, and drug-naive or withdrawn from $\mathrm{D}_{2}$ agonist treatment. Our results show that short term treatment with $\mathrm{D}_{2}$ agonists did not cause any significant changes in the binding of $\left[{ }^{123} \mathrm{I}\right] \beta$-CIT to striatal dopamine transporters. This finding confirms the results of previous animal experiments investigating the influence of several dopamine receptor agonists on the striatal dopamine transporter. ${ }^{13-15}$ Thus measurement of dopaminergic degeneration in the present study was based on the assumption that treatment with $\mathrm{D}_{2}$ agonists or levodopa has no significant influence on $\left[{ }^{123} \mathrm{I}\right] \beta$-CIT binding to dopamine transporters. Nonetheless, all of these studies-including the present one-are limited by small sample sizes (and consequently by limited power), as well as by short duration, and therefore do not exclude the possibility that pharmacological effects could emerge in larger or longer term studies.

Several techniques are now available for imaging nigrostriatal dopaminergic neurones (for a review, see Booij and colleagues $^{29}$ ). In the present study, we used a radiotracer which binds to the dopamine transporter. This transporter is located exclusively on nerve terminals of dopaminergic cells. Several studies have shown that the loss of striatal dopamine transporters in Parkinson's disease is in concordance with the loss of nigrostriatal dopamine neurones. ${ }^{30}$ In addition, necropsy studies of patients with Parkinson's disease have shown that measurements of dopamine transporter density are highly correlated with striatal dopamine levels. ${ }^{31}$ Experimental studies in rats and monkeys have also validated the fact that drug induced losses of nigrostriatal cells are correlated with loss of dopamine transporters, as measured by $\left[{ }^{123} \mathrm{I}\right] /\left[{ }^{125} \mathrm{I}\right] \beta$-CIT. ${ }^{32}{ }^{33}$ Moreover, several $\left[{ }^{123} \mathrm{I}\right] \beta$-CIT SPECT studies have shown loss of dopamine transporters in Parkinson's disease, and correlations between motor signs and severity of loss of these transporters. ${ }^{56}$ These studies have shown the validity of using dopamine transporter imaging to assess the integrity of presynaptic dopaminergic nerve terminals in Parkinson's disease. In fact, $\beta$-CIT SPECT assesses the expression of dopamine transporters on surviving dopaminergic cells. Recently, an important study by Lee et al suggested that the dopamine transporter is downregulated on surviving dopaminergic neurones in Parkinson's disease. ${ }^{34}$ This observation is in line with experimental studies suggesting that the loss of dopamine neurones is functionally compensated by an increase in synthesis and release of dopamine from surviving dopamine nerve terminals, as well as by a reduced rate of dopamine inactivation in presynaptic dopamine nerve terminals. ${ }^{35}$ In the light of all these data, we cannot exclude the possibility in the present study that the loss of dopamine transporters in Parkinson's disease results from a combination of cell loss (and consequently loss of dopamine transporters) and downregulation of dopamine transporters on surviving neurones. However, if the loss of $\beta$-CIT binding to dopamine transporters in Parkinson's disease can be explained partly by downregulation, it seems unlikely that this regulation will disappear, whereas degeneration of dopaminergic neurones 
will progress. Consequently, the possible occurrence of downregulation on surviving dopaminergic nerve terminals in Parkinson's disease may not hamper the applicability of $\left[{ }^{123} \mathrm{I}\right] \beta$-CIT SPECT in monitoring disease progression.

Our study allows sample size calculations for future studies on the evaluation of neuroprotective treatments (table 4). The most extensive deterioration after one year was found in the whole putamen, indicating that in early Parkinson's disease, $\left[{ }^{123} \mathrm{I}\right] \beta$-CIT binding in this region is the most sensitive to changes in the rate of dopaminergic degeneration. We estimated that, in order to show a significant effect of a neuroprotective agent with 0.80 power and $50 \%$ of predicted protection within two years, 78 patients are required in each group when the effects are measured in the whole putamen. For a trial with an agent with $30 \%$ of predicted protection, 216 patients are required. As expected, extending the scanning interval to five years reduces the required sample size remarkably. All estimations are based on the assumption of a constant rate of dopaminergic degeneration through the initial course of the disease. The linear evolution seems a reasonable approximation for the relatively brief period of time involved in the present study. However, if the degeneration shows an exponential course, slowing down in the later disease stages (a reasonable hypothesis), then a larger sample size would be required to detect the same neuroprotective effect.

\section{Conclusions}

Our study shows that $\left[{ }^{123} \mathrm{I}\right] \beta$-CIT SPECT allows measurements of the rate of dopaminergic degeneration in early stage Parkinson's disease. Our observations, in combination with the wide availability of the SPECT technique, makes $\left[{ }^{123} \mathrm{I}\right] \beta$-CIT SPECT an alternative to PET as a method for estimating the effectiveness of putative neuroprotective treatments in large clinical trials. Our study also showed that short term treatment with $\mathrm{D}_{2}$ agonists did not influence $\left[{ }^{123} \mathrm{I}\right] \beta$-CIT binding to dopamine transporters in a small group of patients. If replicated in larger groups of patients, this finding confirms the applicability of $\left[{ }^{123} \mathrm{I}\right] \beta$-CIT SPECT for examining the progression of dopaminergic degeneration in patients who are on these drugs.

\section{ACKNOWLEDGEMENTS}

AW was supported by a grant from NWO-MW, Den Haag, Netherlands.

\section{Authors' affiliations}

A Winogrodzka, P Bergmans, J C Stoof, E C Wolters, Department of Neurology, Vrije Universiteit Medical Centre; Graduate School for Neurosciences, Amsterdam, Netherlands

J Booii, E A van Royen, Department of Nuclear Medicine, Academic Medical Centre, University of Amsterdam

\section{Competing interests: none declared}

\section{REFERENCES}

1 Bernheimer $\mathbf{H}$, Birkmayer W, Hornykiewicz O, et al. Brain dopamine and the syndromes of Parkinson and Huntington. J Neurol Sci 1973;29:415-55.

2 Morrish PK, Sawle GV, Brooks DJ. An [18F]dopa-PET and clinical study of the rate of progression in Parkinson's disease. Brain 1996;119:585-91.

3 Vingerhoets FJG, Snow BJ, Lee CS, et al. Longitudinal fluorodopa positron emission tomographic studies of the evolution of idiopathic parkinsonism. Ann Neurol 1994;36:759-64.

4 Morrish PK, Rakshi JS, Bailey DL, et al. Measuring the rate of progression and estimating the preclinical period of Parkinson's disease with [ $\left.{ }^{18} \mathrm{~F}\right]$ dopa PET. J Neurol Neurosurg Psychiatry 1998;64:314-19.

5 Seibyl JP, Marek KL, Quinlan D, et al. Decreased single-photon emission computed tomographic [ $\left.\left.{ }^{123}\right]\right] \beta$-CIT striatal uptake correlates with symptom severity in Parkinson's disease. Ann Neurol 1995;38:589-98.

6 Tissingh G, Bergmans P, Booii J, et al. Drug-naive patients with Parkinson's disease in Hoehn and Yahr stages I and II show a bilateral decrease in striatal dopamine transporters as revealed by $\left[{ }^{123} \mid\right] \beta-C I T$ SPECT. J Neurol 1998;245: 14-20.

7 Seibyl JP, Marek K, Sheff K, et al. Test/retest reproducibility of iodine-123- $\beta$ CIT SPECT brain measurement of dopamine transporters in Parkinson's disease. J Nucl Med 1997;38:1453-59
8 Brooks DJ. The early diagnosis of Parkinson's disease. Ann Neurol 1998:44:S10-18.

9 Hughes A, Daniel SE, Kilford L, et al. Accuracy of clinical diagnosis of idiopathic Parkinson's disease: a clinico-pathological study of 100 cases. J Neurol Neurosurg Psychiatry 1992:55:181-4.

10 Hoehn MM Yahr MD. Parkinsonism: onset, progression and mortality. Neurology 1967;17:427-42.

11 Fahn S, Elton RL, Members of the UPDRS Development Committee. Unified Parkinson's disease rating scale. In: Fahn S, Marsden CD, Calne $\mathrm{DB}$, et al, eds. Recent developments in Parkinson's disease. Florham Park, NJ: Macmillan Healthcare Information, 1987:153-64.

12 Innis RB, Marek KL, Sheff K, et al. Effect of treatment with L-DOPA/carbidopa or L-selegiline on striatal dopamine transporter SPECT imaging with [ $\left.{ }^{123}\right]$ ] $\beta$-CIT. Move Disord 1999;14:436-42.

13 Vander Borght T, Kilbourn M, Desmond T, et al. The vesicular monoamine transporter is not regulated by dopaminergic drug treatments. Eur J Pharmacol 1995;294:577-83.

14 Dresel SHJ, Kung M-P, Plossl K, et al. Pharmacological effects of dopaminergic drugs on in vivo binding of [99mTc]TRODAT-1 to the central dopamine transporters in rats. Eur J Nucl Med 1988;25:31-9.

15 Little KY, Gorebig J, Carrol Fl, et al. Lack of dopamine receptor agonists effect on striatal dopamine transporter binding sites. Brain Res 1996;742:313-16.

16 Booij J, Tissingh G, Boer GJ, et al. $\left[{ }^{123}\right.$ I]FP-CIT SPECT shows a pronounced decline of striatal dopamine transporter labelling in early and advanced Parkinson's disease. J Neurol Neurosurg Psychiatry 1997:62:133-40.

17 Booij J, Habraken JBA, Bergmans P, et al. Imaging of dopamine transporters with iodine-123-FP-CIT SPECT in healthy controls and patients with Parkinson's disease. J Nucl Med 1998;39:1879-84.

18 Laruelle M, Wallace E, Seibyl JP, et al. Graphical, kinetic and equilibrium analyses of in vivo $\left.\left[{ }^{123}\right]\right] \beta$-CIT binding to dopamine transporters in healthy human subjects. J Cereb Blood Flow Metab 1994; 14:982-94

19 Altman DG. Practical statistics for medical research. London: Chapman and Hall, 1991.

20 Seibyl JP, Innis RB, Early ML, et al. Baseline striatal dopamine transporter uptake measured with [1-123] $\beta$-CIT SPECT may predict the rate of disease progression in idiopathic Parkinson's disease [abstract]. $J$ Nucl Med 1999;40:27P.

21 Parkinson Study Group. Dopamine transporter brain imaging to assess the effects of pramipexole vs levodopa on Parkinson disease progression. JAMA 2002;287:1651-61.

22 Pirker W, Djamshidian S, Asenbaum S, et al. Progression of dopaminergic degeneration in Parkinson's disease and atypical parkinsonism: a longitudinal $\beta$-CIT SPECT study. Mov Disord 2002; 17:45-53.

23 Tissingh G, Bergmans $P$, Booij J, et al. $\left.\left[{ }^{123}\right]\right] \beta-C I T$ single-photon emission tomography in Parkinson's disease reveals a smaller decline in dopamine transporters with age than in controls. Eur J Nucl Med 1997; 24:1171-4.

24 Nurmi E, Ruottinen H, Kaasinen V, et al. Progression in Parkinson's disease: a positron emission tomography study with a dopamine transporter ligand ${ }^{18}$ FCFT. Ann Neurol 2000;47:804-8.

25 Winogrodzka A, Bergmans P, Booii J, et al. [123|]FP-CIT SPECT is a useful method to monitor the rate of dopaminergic degeneration in early-stage Parkinson's disease. J Neural Transm 2001;108:1011-19.

26 Van Dyck CH, Seibyl JP, Malison RT, et al. Age-related decline in striatal dopamine transporter binding with iodine-123-3-CIT SPECT. J Nucl Med 1995; 36:1175-81.

27 Laruelle $M$, Baldwin RM, Malison RT, et al. SPECT imaging of dopamine and serotonin transporters with [ $\left.{ }^{123} \mid\right] \beta$-CIT: pharmacological characterization of brain uptake in nonhuman primates. Synapse 1993;13:295-309.

28 Ahlskog JE, Uitti RJ, O'Connor MK, et al. The effect of dopamine agonist therapy on dopamine transporter imaging in Parkinson's disease. Mov Disord 1999; 14:940-6.

29 Booij J, Tissingh G, Winogrodzka A, et al. Imaging of the dopaminergic neurotransmission system using single-photon emission tomography and positron emission tomography in patients with parkinsonism [review]. Eur J Nucl Med 1999:26:171-82.

30 Kaufman MJ, Madras BK. Severe depletion of cocaine recognition sites associated with the dopamine transporter in Parkinson's-diseased striatum. Synapse 1991;9:43-9.

31 Guttman M, Burkholder J, Kish SJ, et al. [ $\left.{ }^{11} \mathrm{C}\right] \mathrm{RTI}-32$ PET studies of the dopamine transporter in early dopa-naive Parkinson's disease: implications for the symptomatic threshold. Neurology 1997;48: 1578-83.

32 Shaya EK, Scheffel U, Dannals RF, et al. In vivo imaging of dopamine reuptake sites in the primate brain using single photon emission computed tomography (SPECT) and iodine-123 labeled RTI-55. Synapse 1992;10:169-72.

33 Ito $Y$, Fujita $M$, Shimada S, et al. Comparison between the decrease of dopamine transporter and that of L-DOPA uptake for detection of early to advanced stage of Parkinson's disease in animal models. Synapse 1999:31:178-85.

34 Lee CS, Samii A, Sossie V, et al. In vivo positron emission tomographic evidence for compensatory changes in presynaptic dopaminergic nerve terminals in Parkinson's disease. Ann Neurol 2000;47:493-503.

35 Zigmond MJ, Abercrombie ED, Berger TW, et al. Comparison after lesions of central dopaminergic neurons: some clinical and basic implications. Trends Neurosci 1990;13:290-6. 